\title{
HÁ ASSOCIAÇÃO ENTRE EXPERIÊNCIA COLETIVA E PRODUTIVIDADE NA PRESTAÇÃO DE SERVIÇOS MEDIADA POR TEMPO, EQUIVALÊNCIA E CUSTOS?
}

\author{
IS THERE AN ASSOCIATION BETWEENCOLLECTIVE EXPERIENCE AND \\ PRODUCTIVITY IN THE PROVISION OF SERVICES MEDIATED BY TIME, \\ EQUIVALENCE AND COSTS?
}

\author{
Mara Juliana Ferrari ${ }^{1}$ \\ Centro Universitário para o Desenvolvimento \\ do Alto Vale do Itajaí (UNIDAVI) \\ mara@unidavi.edu.br
}

\author{
Altair Borgert \\ Universidade Federal de Santa Catarina \\ (UFSC) \\ altair@borgert.com.br
}

\begin{abstract}
RESUMO
Esta pesquisa objetivou verificar se há associação entre experiência coletiva e produtividade na prestação de serviços. Caracterizado por um estudo de caso, predominantemente quantitativo e de natureza exploratória e descritiva, utilizou-se dados de produção por meio de equivalência, custos e tempo de permanência de integrantes de 11 equipes de trabalho de uma empresa prestadora de serviços de telecomunicações, ao longo de 24 meses. Para tanto, formulou-se duas hipóteses de pesquisa: hipótese nula, que não existe associação entre a variável Experiência e Produtividade ao longo do período considerando-se o tempo médio das equipes e, como hipótese alternativa, que as variáveis são associadas. Como resultado, por meio do teste Qui-quadrado $\left(\chi^{2}\right)$ obteve-se o valor de 3,7161, ao nível de significância de $\alpha=0,05$, com 4 graus de liberdade, e valor crítico de 9,48773 que representa a área caudal direita. Assim, como o valor Qui-quadrado está fora da área aceitável, não se pode rejeitar a hipótese nula, o que leva a conclusão de que os dados não mostram evidência de associação entre Experiência e Produtividade, para o caso estudado, cujas diferenças nas proporções podem ser explicadas por simples variações causais.
\end{abstract}

Palavras-chave: Experiência coletiva. Produtividade. Equivalência e custos.

\section{ABSTRACT}

This research aimed to verify if there is an association between collective experience and productivity in the provision of services. Characterized by a case study, predominantly quantitative and of an exploratory and descriptive nature, production data were used through equivalence, costs and length of stay of members of 11 work teams of a company providing telecommunications services, throughout 24 months. For this, two research hypotheses were formulated: the null hypothesis, that there is no association between the variable Experience

${ }^{1}$ Rua Dr. Guilherme Gemballa, 13 - Jardim América, CEP: 89160-932 - Rio do Sul - SC. 
and Productivity over the period considering the average time of the teams and, as an alternative hypothesis, that the variables are associated. As a result, by means of the Chi-square test $\left(\chi^{2}\right)$ a value of 3.7161 was obtained, at a significance level of $\alpha=0.05$, with 4 degrees of freedom, and with a critical value of 9.48773 representing the right caudal area. Thus, since the Chi-square value is outside the acceptable area, the null hypothesis cannot be rejected, which leads to the conclusion of. Thus, it can be concluded that the data show no evidence of an association between Experience and Productivity, for the case studied, whose differences in proportions can be explained by simple causal variations.

Keywords: Collective experience. Productivity. Equivalence and costs.

\section{INTRODUÇÃO}

Empresas prestadoras de serviços têm como característica o uso intensivo da mão de obra, cujos profissionais utilizam o conhecimento, as habilidades e a experiência adquirida ao longo do tempo por meio da repetição, além de outros fatores, para a execução das suas atividades. Em decorrência, surge o efeito da aprendizagem ao longo do processo, o que implica em mais conhecimentos, mais habilidades ou em experiência acumulada, resultando no aumento da produtividade (Wright, 1936; Adler \& Clark, 1991; Teplitz, 1991; Badiru, 1992; Argote, 1999; Fioretti, 2007).

Em termos funcionais, para o emprego da expressão "conhecimento" no âmbito organizacional, Davenport e Prusak (2000) o definem como uma mistura de experiência condensada, valores, informação e insight experimentado no qual se incorporam novas experiências e informações. E, acrescentam que o conhecimento está embutido não só em documentos ou repositórios, mas, também, em rotinas, processos, práticas e normas. Nessa direção, Sveiby (1998) chama a atenção para o termo "competência" que é constituído por cinco elementos mutuamente dependentes: a) conhecimento explícito - adquirido por meio de informações; b) habilidades - que é a arte de saber fazer, adquirida por meio da própria prática e do treinamento; c) experiência - reflexão sobre acertos e erros passados; d) julgamentos de valor - percepções consideradas corretas; e e) rede social - constituída pelas relações com os outros no interior de um ambiente.

Contudo, a ambiguidade atribuída aos termos "conhecimento" e "competência" remete ao direcionamento de uma nova definição - o conceito de experiência - quanto ao aspecto organizacional. Assim, os elementos apresentados por Sveiby (1998) e Davenport e Prusak (2000), alguns em maior escala do que outros, conjuntamente, representam o que se pode chamar de "experiência". Além disso, no contexto do trabalho coletivo surge o conceito de "sinergia" que, segundo Corning (1983), consiste no esforço coordenado de vários subsistemas para a realização de uma tarefa complexa ou função de determinado projeto. E, é neste contexto que o presente trabalho se insere, na medida em que estabelece o relacionamento entre aspectos complementares como a produtividade resultante, também, da experiência coletiva.

A experiência adquirida por meio da repetição é um elemento importante para a análise da produtividade empresarial. Ou seja, quanto mais vezes uma tarefa ou função é repetida por um profissional - ou uma equipe - mais experiente este se torna, e menor tende a ser o dispêndio de tempo para a sua execução. Em geral, quanto maior o nível de conhecimento dos funcionários acerca do que realizam, maior tende a ser o resultado do seu trabalho (Wright, 1936; Dutton \& Thomas, 1984; Adler \& Clarck, 1991; Teplitz, 1991; Badiru, 1992; Argote, 1999; Boone \& Ganeshan, 2001; Argote, Mcevily, \& Reagans, 2003; Fioretti, 2007; Lohmann et al., 2019).

Para medir o fenômeno da experiência, como resultado da aprendizagem adquirida por meio de processos repetitivos, e prever o tempo para a execução das atividades, os gestores 
utilizam modelos de curvas de aprendizagem que possibilitam verificar como as horas por unidade diminuem na medida em que a produção aumenta. Assim, são pilares para fins de análise a aprendizagem, a competência e o aperfeiçoamento das pessoas que pertencem às equipes de trabalho nas suas respectivas funções (Horngren, Datar, \& Foster, 2004).

Devido as suas características, geralmente organizações prestadoras de serviços planejam, executam e controlam os projetos ou obras por meio de equipes de trabalho. Num processo sinergético, executam as obras e buscam cumprir as metas pré-estabelecidas, cuja produção é medida no seu todo e a produtividade depende dos recursos alocados ao grupo. Assim, para uma análise coletiva da produção utilizam-se elementos que podem, individual ou conjuntamente, explicar os fenômenos observados num processo produtivo. Nesta direção, Huckman, Staats e Upton (2009) consideraram o número de projetos desenvolvidos pelas equipes como medida para o total da produção e, para medir a experiência, o tempo despendido em cada projeto pelos seus integrantes.

Para o desenvolvimento conjunto de atividades na prestação de serviços, algumas empresas utilizam unidades de medida, baseadas em equivalência, para fins de representação coletiva da produção. Comumente, tais empresas são dotadas de características multiprodutoras e apresentam certa complexidade no processo de alocação dos custos, cujos modelos com base em equivalência se apresentam como alternativa para solucionar tal problemática (De La Villarmois \& Levant, 2007; Gervais \& Levant, 2007; Bornia, 2010; Levant \& De La Villarmois, 2011; Levant \& Zimnowitch, 2013). Neste sentido, vale citar os trabalhos de Ferrari (2012) e Reis, Borgert e Ferrari (2016) aplicados a uma empresa prestadora de serviços do setor de telecomunicações, em que analisaram a relação entre os custos e a produção de equipes de trabalho de diversas categorias e concluíram que há correlação moderada entre as variáveis estudadas, o que permite a utilização dos mesmos dados para a verificação da produtividade neste artigo.

Diante dos resultados apresentados por Ferrari (2012) e Reis, Borgert e Ferrari (2016), infere-se que outras variáveis podem influenciar no comportamento da produtividade, como o tempo de experiência dos integrantes das equipes, por exemplo. Talvez, não exista uma relação direta entre tais elementos, devido a existência de um conjunto de fatores que influenciam na aprendizagem dos indivíduos, bem como na sinergia das equipes. Mas, é possível o estabelecimento de algum grau de associação entre tais elementos.

Assim, na presente pesquisa, leva-se em consideração a experiência dos indivíduos que formam uma equipe de trabalho sob o aspecto da arte de saber fazer e "aprender-fazendo". Este é um tipo de aprendizagem defendido por Adler e Clark (1991) que resulta em melhorias da produtividade por meio da tarefa de repetição. Além disso, os autores consideram a experiência como geração incremental, adquirida por meio da própria prática e do treinamento que, em geral, é consequência do tempo médio de permanência dos integrantes nas equipes de trabalho. Em função do exposto, o presente estudo busca responder o seguinte questionamento: a experiência coletiva tem associação com a produtividade na prestação de serviços? E, para responder à questão central, esta pesquisa tem como objetivo verificar se há associação entre experiência coletiva e produtividade na prestação de serviços.

A relevância do presente trabalho, sob a ótica gerencial, quanto ao caráter repetitivo em empresas prestadoras de serviços, pode ser considerada por alguns fatores como: a) o conhecimento da produtividade das equipes - em cada projeto ou obra - é importante para estimar os custos futuros e, assim, oferecer parâmetros para o planejamento da organização; e b) os índices de produtividade podem auxiliar na melhoria dos processos produtivos.

Por fim, sob a ótica acadêmica, a presente pesquisa contribui para os estudos que abordam a aprendizagem de forma coletiva, uma vez que, regra geral, as pesquisas utilizam a abordagem individual de aprendizagem. Além disso, aprofunda as pesquisas realizadas sobre as informações derivadas da permanência de um indivíduo numa mesma equipe de trabalho 
durante o processo produtivo. Mais especificamente, estima-se o efeito desta variável quanto ao esforço de produção e o uso de tal medida na compreensão e contribuição dos índices de produtividade, de modo a oferecer um avanço conceitual no âmbito gerencial.

\section{FUNDAMENTAÇÃO TEÓRICA}

A produtividade está relacionada com a eficiência de um processo ou o melhor uso dos recursos como a matéria-prima, equipamentos, energia, capital, além de outras variáveis econômicas e ambientais para a produção de atividades. Segundo Colares (2005), com os estudos de Elton Mayo, fatores psicológicos foram, também, atribuídos ao rol de variáveis que interferem nos índices de produtividade de indivíduos e equipes, dentre os quais o relacionamento social entre operários e supervisores, a satisfação e a insatisfação com as tarefas realizadas, a organização informal que afeta mais os resultados de produção que a formal e o comportamento individual que é fortemente afetado pelas diretrizes estabelecidas pelo grupo.

Nessa direção, Antonello e Godoy (2010) enfatizam que os estudos sobre aprendizagem organizacional têm avançado em termos de volume de publicações e, também, na dimensão do tema para além da gestão propriamente dita, o que leva a vários questionamentos e sugerem a adoção de uma perspectiva baseada em práticas. De acordo com Glock et al. (2019), a literatura em aprendizagem organizacional e, em especial, sobre curvas de aprendizagem é vasta. Stroieke, Fogliatto e Anzanello (2013) atribuem essa condição ao fato de que tal metodologia é utilizada em vários segmentos e com propósitos diversos, como o de estimar o tempo de conclusão de um produto, avaliar a produção na redução de custos, otimizar a atribuição de tarefas aos trabalhadores baseados em seu perfil de aprendizagem e suavizar as perdas de produção após a interrupção das tarefas.

No atual mercado há exigência cada vez maior de técnicas e ferramentas de gestão para auxílio a informações relativas à gestão dos custos e gerenciamento dos processos e avaliação de índices de produtividade. Uma importante informação gerencial, na área da estratégia competitiva, é a medida que identifica o quanto a aprendizagem dos trabalhadores influencia os processos, identificada como curvas de aprendizagem (Demeester \& Qi, 2005). A indústria de montagem de aviões foi a primeira a documentar o efeito que a aprendizagem tem sobre a eficiência (Horgren et al., 2004), em que a análise de custos foi o fator gerador do estudo sobre aprendizado, o qual foi denominado de "Curvas de Aprendizagem". Vale destacar que Wright (1936) desenvolveu o primeiro modelo de curva de aprendizagem para demonstrar a redução nos custos de produção numa planta de pequenos aviões (Wiersma, 2007; Morrison, 2008). Contudo, segundo Morrison (2008), o conceito já se aplicava a Adam Smith, que buscou descrever um exemplo de pino de tomada como uma ilustração de aprendizagem baseada na experiência.

A curva de aprendizagem é uma descrição matemática do desempenho dos trabalhadores em tarefas repetitivas (Wright, 1936; Teplitz, 1991; Badiru, 1992; Argote, 1999; Fioretti, 2007). No entanto, Togo (2001) considera a curva de aprendizagem uma empírica relação entre grandezas de recursos e horas de trabalho consumidas, e classifica como um aspecto importante da estimativa de custos gerencial. Em seus estudos, apresenta o caso da empresa Akamai Missile com o propósito de ilustrar uma análise realista da aplicação de curvas de aprendizagem e seu impacto na estimativa dos custos, cujo objetivo central era demonstrar a aplicação de uma variedade de funções, tais como polinomial, média exponencial e logarítmica em contrapartida aos modelos lineares apresentados pelos livros de custos. Tal argumentação é defendida pela autora por trazer dados mais precisos aos cálculos de curvas de aprendizagem que os modelos lineares usados até então.

Os estudos de Anzanello e Fogliatto (2011) e Stroieke, Fogliatto e Anzanello (2013) apresentaram o estado da arte da literatura sobre curvas de aprendizagem, em que descreveram 
os modelos existentes e suas limitações, bem como relataram as suas aplicações nos mais diversos segmentos e apresentaram proposições para futuras pesquisas sobre o assunto. Para Anzanello e Fogliatto (2011), a variedade de aplicações justifica o grande número de publicações sobre o assunto. Concluem que a maioria das proposições são motivadas pela crescente popularidade de customização em massa em indústrias e serviços, e pelos benefícios que podem retirar dos modelos de curva de aprendizagem. Além disso, sugerem a exploração do uso de curvas de aprendizagem em modelos multivariados, visto serem pouco investigados na literatura.

Stroieke, Fogliatto e Anzanello (2013) sugeriram estudos com a abordagem da aprendizagem coletiva, visto que na maioria das pesquisas utiliza-se o processo individual de aprendizado. Podem utilizar a curva de aprendizagem para a criação de agrupamentos homogêneos de trabalhadores, ou estudar como o processo de aprendizado contribui para a definição de estratégias da empresa por afetar diretamente os custos de produção. Além disso, classificam como monitoramento de custos os estudos de Alchian (1963), Conley (1970), Jaber e Bonney (1996), Szwarcfiter e Dalcol (1997), Leite (2002), Pereira e Suslick (2003), Smunt e Watts (2003), Tsuchiya e Kobayashi (2004), Nemet (2006), Weitzel, Rovere e Cunha (2006), Neuhaus e Bunke (2007), Kahouli-Brahmi (2008), Morrison (2008), Plaza e Rohlf (2008), Young, Masel e Judd (2008), Van Den Wall Bake et al. (2009), Van Den Broek et al. (2009), De Wit et al. (2010), Nadeau et al. (2010) e Schoots, Kramer e Van Der Zwaan (2010) que utilizaram, simultaneamente, a curva de aprendizagem e foram efetuados nos mais variados segmentos organizacionais.

Vale destaque para alguns autores, como Nadeau et al. (2010), que apresentam um quadro analítico que possibilita aos tomadores de decisão incorporarem informações sobre a evolução esperada em suas avaliações econômicas de tecnologia com a utilização da modelagem de custo baseada no processo. Argumentam que esta modelagem fornece uma estrutura conveniente dentro da qual estudam o impacto da aprendizagem sobre os principais condutores dos custos. Ainda, exploram o valor desta abordagem e examinam o efeito da aprendizagem em alguns parâmetros do processo, tais como tempo de ciclo, tempo de inatividade e taxa de rejeição sobre a evolução dos custos, a qual fornece uma compreensão do nível técnico de como a evolução dos custos depende das características do produto ou processo. Concluem que a teoria da aprendizagem fornece um quadro útil para examinar os ganhos de produtividade que se acumulam ao longo do tempo, com o aumento da experiência.

Por outro lado, Wiersma (2007) examinou fatores que identificam o aumento da capacidade e a oportunidade de aprender, e discute a hipótese de que a capacidade de aprender é reforçada pela presença de uma quantidade moderada de empregados temporários no mercado de trabalho, o que proporciona aos funcionários variação das tarefas, medida pela heterogeneidade do produto. Conclui que o aumento da capacidade e a oportunidade de aprender leva aos benefícios esperados com empregados temporários. No entanto, os processos que conduzem a essa aprendizagem não são bem compreendidos, bem como há dificuldade, com base no estudo, para sustentar tal afirmação. Uma possibilidade é que um grupo diverso tem maior capacidade de alinhar conhecimento velho com o novo. Para a autora, quando as firmas estão na fase inicial da curva de aprendizagem, o aprendizado pode ser maior em comparação com membros que já compõem as equipes.

Ainda, Boone e Ganeshan (2001) direcionaram suas pesquisas para a análise da relação entre a experiência e a produtividade num período de 10 anos de uma organização de serviço profissional na área de engenharia. Segundo os autores, a pesquisa explorou uma lacuna na literatura no que diz respeito aos modelos organizacionais quanto a experiência em empresas prestadoras de serviços. Analisaram, primeiramente, a relação entre a experiência de produção e a produtividade e, em seguida, investigaram o efeito que a experiência com informações advindas do uso da tecnologia tem sobre essa relação. Para tanto, definiram como variável 
dependente a produtividade organizacional, para a qual atribuíram o número de projetos concluídos durante um período de tempo específico o que é uma situação análoga ao número de unidades produzidas num ambiente de fabricação. Concluíram que há uma relação significativa e positiva entre experiência organizacional e produtividade medida pelo número de projetos numa prestadora de serviços.

Por fim, Huckman, Staats e Upton (2009) desenvolveram uma pesquisa sobre o aprendizado em equipes. Justificaram tal estudo pelo fato de que grande parte da literatura trata a experiência como um conceito unidimensional, capturado pelo volume acumulado de produção, ou o número de projetos concluídos por uma equipe, bem como pressupõe que as equipes são estáveis em sua composição e organização interna. Para os autores, tal estabilidade é rara porque a composição e estrutura das equipes frequentemente mudam ao longo do tempo. Assim, o ambiente da pesquisa e da coleta de dados se deu em uma empresa de serviços de software indiana com o propósito de examinar como as mudanças, de membros nas equipes, podem afetar o acúmulo de experiência e o desempenho. Os autores identificaram que o nível de familiaridade da equipe (ou seja, o número médio de vezes que cada membro tem trabalhado com todos os outros membros da equipe) tem um efeito positivo significativo sobre o desempenho.

Contudo, apesar de algumas considerações sobre a temática realizadas por Ostermeier (2019), ao abordar as questões humanas em produção diversificada, as medidas convencionais da experiência de cada membro da equipe como, por exemplo, anos na empresa, não são consistentemente relacionadas com as performances.

A Tabela 1 apresenta um resumo da literatura discutida até o momento, com destaque para os autores e foco/objetivo de cada pesquisa.

Tabela 1. Resumo da literatura referenciada

\begin{tabular}{|c|c|}
\hline Autores & Foco/objetivo da pesquisa \\
\hline Wright (1936). & Primeiro modelo de curva de aprendizagem com descrição matemática. \\
\hline $\begin{array}{l}\text { Alchian (1963); Conley (1970); Jaber } \\
\text { e Bonney (1996); Szwarcfiter e Dalcol } \\
\text { (1997); Leite (2002); Pereira e Suslick } \\
\text { (2003); Smunt e Watts (2003); } \\
\text { Tsuchiya e Kobayashi (2004); Nemet } \\
\text { (2006); Weitzel, Rovere e Cunha } \\
\text { (2006); Neuhaus e Bunke (2007); } \\
\text { Kahouli-Brahmi (2008); Plaza e Rohlf } \\
\text { (2008); Young, Masel e Judd (2008); } \\
\text { Van Den Wall Bake et al. (2009); Van } \\
\text { Den Broek et al. (2009); De Wit et al. } \\
\text { (2010); Nadeau et al. (2010); Schoots, } \\
\text { Kramer e Van Der Zwaan (2010). }\end{array}$ & $\begin{array}{l}\text { Monitoramento dos custos de produção por meio da curva de } \\
\text { aprendizagem. }\end{array}$ \\
\hline $\begin{array}{l}\text { Teplitz (1991); Badiru (1992); Argote } \\
\text { (1999); Fioretti (2007). }\end{array}$ & $\begin{array}{l}\text { Curva de aprendizagem é uma descrição matemática do desempenho } \\
\text { dos trabalhadores em tarefas repetitivas. }\end{array}$ \\
\hline Togo (2001). & $\begin{array}{l}\text { Curva de aprendizagem classificada como um aspecto importante da } \\
\text { estimativa de custos gerencial. }\end{array}$ \\
\hline Boone e Ganeshan (2001). & $\begin{array}{l}\text { Lacuna de estudos em empresas prestadoras de serviços, como a relação } \\
\text { entre experiência organizacional e produtividade medida pelo número } \\
\text { de projetos numa prestadora de serviços. }\end{array}$ \\
\hline Horgren et al. (2004). & $\begin{array}{l}\text { A indústria de montagem de aviões foi a primeira a documentar o efeito } \\
\text { que a aprendizagem tem sobre a eficiência. }\end{array}$ \\
\hline Colares (2005). & $\begin{array}{l}\text { Fatores psicológicos interferem nos índices de produtividade de } \\
\text { indivíduos e equipes. }\end{array}$ \\
\hline Demeester e Qi (2005). & Influência da aprendizagem dos trabalhadores nos processos. \\
\hline Wersma (2007). & $\begin{array}{l}\text { Quando as firmas estão na fase inicial da curva de aprendizagem, o } \\
\text { aprendizado pode ser maior com membros que já compõem as equipes. }\end{array}$ \\
\hline
\end{tabular}




\begin{tabular}{|l|l|}
\hline Morrison (2008). & $\begin{array}{l}\text { Atribui a Adam Smith o uso de curva de aprendizagem por meio de uma } \\
\text { ilustração de aprendizagem com base na experiência. }\end{array}$ \\
\hline Huckman, Staats e Upton (2009). & O nível de familiaridade da equipe tem efeito sobre o desempenho. \\
\hline Nadeau et al. (2010). & $\begin{array}{l}\text { A teoria da aprendizagem fornece um quadro útil para examinar os } \\
\text { ganhos de produtividade com o aumento da experiência. }\end{array}$ \\
\hline Antonello e Godoy (2010). & Avanço dos estudos sobre aprendizagem organizacional. \\
\hline Anzanello e Fogliatto (2011). & $\begin{array}{l}\text { Proposições para futuras pesquisas em curvas de aprendizagens nas } \\
\text { organizaçes. }\end{array}$ \\
\hline $\begin{array}{l}\text { Stroieke, Fogliatto e Anzanello } \\
(2013) .\end{array}$ & $\begin{array}{l}\text { Estado da arte sobre o tema e proposições para pesquisas com } \\
\text { abordagem na aprendizagem coletiva. }\end{array}$ \\
\hline Glock et al. (2019). & Literatura em aprendizagem organizacional e curvas de aprendizagem. \\
\hline Ostermeier (2019). & $\begin{array}{l}\text { Medidas convencionais da experiência de cada membro da equipe não } \\
\text { são consistentemente relacionadas com as performances. }\end{array}$ \\
\hline
\end{tabular}

Fonte: Dados da pesquisa

Portanto, com base no referencial apresentado, pode-se discutir os aspectos metodológicos inerentes à presente pesquisa.

\section{ASPECTOS METODOLÓGICOS}

Quanto ao objetivo, a presente pesquisa é de natureza exploratória e descritiva, caracterizada por um estudo de caso. Busca-se resposta para a questão levantada, por meio da associação entre variáveis, no sentido de explicar um fenômeno específico para um caso empresarial em que se aplica uma unidade de medida de produção baseada em equivalência e utilizada para o gerenciamento dos custos e da produção de equipes trabalho.

A perspectiva de análise da pesquisa é longitudinal, com dois cortes transversais. $\mathrm{Ou}$ seja, utilizam-se informações referentes ao período de 02 (dois) anos de trabalho de equipes de prestação de serviços da empresa objeto do estudo. A abordagem do problema tem característica predominantemente quantitativa, em que se procura na estatística descritiva a análise de teste de hipóteses por meio do Teste Qui-quadrado, que tem por função principal a comparação das frequências observadas com as esperadas em cada categoria (Fávero et al., 2009), e meios para o alcance do objetivo geral, qual seja a associação entre as variáveis produtividade e experiência. Além disso, utilizam-se os programas Excel®, para a organização dos dados e tabelas, e o pacote estatístico Stata ${ }^{\circledR}$ e Gretl ${ }^{\circledR}$, para análises de associação e $p$-value.

A unidade de observação deste estudo compreende uma organização de grande porte que realiza a prestação de serviços na área de telecomunicações, dentre os quais o acesso a rede pública, o provimento de conexões e de conteúdo, o estabelecimento de transmissão e, em especial, a implantação de redes telefônicas. Assim, o levantamento de dados se baseia em registros institucionais e relatórios gerenciais de uma empresa do setor telecomunicações, que tem por característica a execução de diversas atividades para a realização de obras de telefonia por meio de equipes de trabalho classificadas como de Classe L, com atividades e funções específicas. No entanto, com o intuito de manter o sigilo sobre os dados aqui apresentados, o nome da empresa e o seu endereço não são divulgados.

\subsection{Definição das variáveis}

Para um melhor entendimento do processo de análise dos dados, e em resposta ao problema central da pesquisa, torna-se necessária a definição das principais variáveis envolvidas no contexto deste trabalho.

- Experiência - obtida pelo tempo de operação de cada equipe em registros de admissão dos seus integrantes de acordo com a data da respectiva produção. Após o levantamento dos tempos de cada integrante, aplicou-se a média dos mesmos como 
medida central representativa de cada equipe.

- Produtividade - obtida pela razão entre "produção" e "custos" em cada período produtivo. A "produção" é representada pela soma das unidades equivalentes de produção das diversas atividades realizadas num mês por uma equipe de trabalho, enquanto que os "custos" representam a força de trabalho de uma equipe, formados pela soma dos salários mensais, seus encargos e benefícios.

Diante do exposto, deseja-se verificar se os dados mostram evidências suficientes para afirmar que nas equipes objeto do estudo, que prestam serviços diversificados e cuja produção é medida por meio de unidades de equivalência, existe associação entre a variável "Experiência" (medida pelo tempo médio de permanência dos membros nas equipes) e a variável "Produtividade" (medida pela razão Produção/Custo). Ou seja, se o aumento (ou diminuição) da experiência interfere na produtividade das equipes de trabalho, o que possibilita formular a seguinte hipótese nula:

- $H_{0}$ : Experiência e Produtividade são variáveis independentes nas equipes estudadas ao longo do período.

Logo, se a experiência das equipes aumenta (ou diminui) há uma associação com a produtividade das equipes. Desta forma, formula-se a seguinte hipótese alternativa:

- $H_{1}$ : Há associação entre as variáveis Experiência e Produtividade nas equipes estudadas ao longo do período.

Assim, as hipóteses formuladas viabilizam as análises dos resultados para o caso em questão levando-se em consideração, também, o referencial teórico.

\section{ANÁLISE DOS RESULTADOS}

A temática em discussão foca na possível associação entre a experiência e a produtividade para equipes de trabalho classificadas na classe L, numa empresa prestadora de serviços de telecomunicações. Para tal, necessário se faz a descrição dos dados com aplicação das respectivas técnicas estatísticas.

\subsection{Fonte e levantamento dos dados}

A empresa, objeto do estudo, contava com diversas equipes para a execução das atividades de telecomunicações para as atividades enquadradas na classe L. Vale destacar que tais atividades, para efeitos de classificação interna da empresa, dizem respeito basicamente aos serviços de instalação de cabos telefônicos e demais serviços auxiliares necessários à sua fixação como colocação de postes, dutos, braçadeiras e aterramento. Ao todo, a empresa operava com 11 equipes nominadas e numeradas com a seguinte formatação: L001, L002, L003, L004, L005, L006, L007, L008, L009, L021 e L022, cujos dados compõem os custos das equipes (medidos em $\mathrm{R} \$$ ), as produções (medidas em unidades equivalentes) e o tempo (medido pelo número de dias que o integrante da equipe permaneceu desde a sua admissão até a data da produção ou saída da equipe).

Para o desenvolvimento das diversas atividades relacionadas a classe L, a empresa segue um padrão para a formação de equipes de trabalho, considerando-se o padrão pelas seguintes funções e quantidades de funcionários: a) Encarregado de Obras (01); b) Encarregado de Classe (01); c) Linheiro (02); e d) Ajudante (03). As várias funções se complementam num processo de sinergia, tendo em vista as especificidades das atividades desenvolvidas. Todavia, este padrão é apenas uma recomendação técnica da empresa, com base em estudos da sua engenharia de produção, mas que pode ser alterado. Assim, de acordo com as necessidades das obras, uma equipe em campo pode apresentar uma configuração diferente, tanto em termos de composição 
numérica, quanto de funções técnicas. Contudo, por meio dos relatórios fornecidos pela empresa, observa-se que normalmente uma equipe de classe $\mathrm{L}$ é formada por, aproximadamente, 07 (sete) funcionários em diversas funções, conforme descrito.

Após levantamento dos dados e composições das equipes foram verificados os custos correspondentes a cada período, bem como a produção apresentada em unidades equivalentes, o que possibilitou somar as várias atividades executadas. De posse desses dados, identificou-se os períodos produtivos e, para a definição da produtividade, dividiu-se a produção de cada período pelo custo correspondente que resultou na razão produção/custo de 11 equipes para um total de 193 observações. Vale lembrar que em alguns meses, durante o período da análise, algumas equipes não registraram produção.

Quanto ao levantamento dos tempos, para cada integrante das equipes, o mesmo se deu por meio dos relatórios fornecidos pela empresa. Assim, verificou-se a alocação de cada membro e sua admissão e identificou-se o tempo no respectivo mês de produção de cada integrante. Após esta constatação, somou-se todos os tempos e aplicou-se a média com o propósito de se utilizar uma medida central em relação ao tempo de permanência na equipe.

Para fins de ilustração, a Tabela 2 apresenta a disposição dos dados, ao longo do período, para duas equipes (L001 e L005), cujos mesmos procedimentos foram efetuados, também, para as demais equipes do estudo.

Tabela 2. Dados de custo, produção e tempo das equipes L001 e L005

\begin{tabular}{c|ccc|ccc}
\hline MÊS & L001C & L001P & L001TM & L005C & L005P & L005TM \\
\hline $\mathbf{1}$ & - & - & - & $7.241,24$ & $2.193,31$ & 61,00 \\
$\mathbf{2}$ & - & - & - & $6.423,65$ & $1.094,40$ & 91,00 \\
$\mathbf{3}$ & $6.135,46$ & 496,00 & 16,00 & - & - & - \\
$\mathbf{4}$ & $7.352,41$ & $2.048,94$ & 48,00 & - & - & - \\
$\mathbf{5}$ & $7.419,99$ & $2.009,36$ & 78,00 & $7.148,47$ & $2.293,89$ & 183,00 \\
$\mathbf{6}$ & $7.112,18$ & $1.979,72$ & 109,00 & $6.471,15$ & 918,91 & 214,00 \\
$\mathbf{7}$ & $7.190,40$ & $2.363,28$ & 139,00 & $6.341,26$ & 958,06 & 244,00 \\
$\mathbf{8}$ & $5.593,11$ & $1.007,75$ & 157,50 & $7.045,11$ & $2.128,91$ & 275,00 \\
$\mathbf{9}$ & $7.114,85$ & $2.125,54$ & 186,00 & $6.720,01$ & $2.686,81$ & 306,00 \\
$\mathbf{1 0}$ & $6.739,65$ & $1.391,51$ & 214,00 & $5.941,95$ & $1.834,31$ & 334,00 \\
$\mathbf{1 1}$ & $6.795,27$ & $1.208,71$ & 231,00 & $6.427,21$ & $1.963,75$ & 365,00 \\
$\mathbf{1 2}$ & $7.545,70$ & $2.951,25$ & 275,00 & $6.114,43$ & $1.662,95$ & 395,00 \\
$\mathbf{1 3}$ & $6.979,90$ & $3.023,29$ & 320,00 & $6.835,72$ & $2.095,35$ & 27,00 \\
$\mathbf{1 4}$ & $7.226,26$ & $2.002,16$ & 263,00 & $6.558,39$ & $1.317,38$ & 57,00 \\
$\mathbf{1 5}$ & $7.369,09$ & $2.404,88$ & 294,00 & $7.302,11$ & $1.616,72$ & 88,00 \\
$\mathbf{1 6}$ & $7.583,51$ & $1.886,56$ & 178,50 & $7.020,89$ & $1.828,65$ & 98,00 \\
$\mathbf{1 7}$ & $10.251,88$ & $5.064,98$ & 208,50 & $6.968,86$ & $1.591,86$ & 128,00 \\
$\mathbf{1 8}$ & $8.879,20$ & $2.655,55$ & 239,50 & $7.182,64$ & $2.573,32$ & 159,00 \\
$\mathbf{1 9}$ & $7.473,92$ & $1.802,06$ & 329,00 & $5.293,94$ & 308,00 & 126,00 \\
$\mathbf{2 0}$ & $7.202,88$ & $1.039,97$ & 360,00 & $6.060,13$ & $1.461,81$ & 43,00 \\
$\mathbf{2 1}$ & $6.789,60$ & $1.785,24$ & 472,00 & - & - & - \\
$\mathbf{2 2}$ & - & - & - & $5.566,73$ & 663,02 & 97,00 \\
$\mathbf{2 3}$ & $6.256,56$ & $2.109,91$ & 624,00 & $6.429,26$ & $1.297,85$ & 128,00 \\
$\mathbf{2 4}$ & $7.041,19$ & $1.847,80$ & 495,50 & $6.069,81$ & $1.916,56$ & 158,00 \\
\hline
\end{tabular}

Legenda: L001C = Custo em R \$ da equipe L001; L001P = Produção em unidades equivalentes da equipe L001; e L001TM = Tempo médio de permanência dos integrantes na equipe L001.

Fonte: Dados da pesquisa

Diante da exposição dos dados referenciados na Tabela 2, e por meio da estatística descritiva, com auxílio do software $S_{t a t a}{ }^{R}$, infere-se algumas observações em relação às variáveis produtividade e experiência, conforme se apresenta na Figura 1. 


\begin{tabular}{|c|c|c|c|c|c|}
\hline Variable & $\mathrm{Obs}$ & Mean & Std. Dev. & Min & $\operatorname{Max}$ \\
\hline produtivid e & 193 & .2455027 & .0975936 & .0488666 & .6392344 \\
\hline experiencia & 193 & 165.8005 & 120.5626 & 16 & 624 \\
\hline
\end{tabular}

Figura 1. Valores de saída do Stata para as 193 observações

Fonte: Dados da pesquisa

Conforme exposto, as 193 observações da variável Produtividade apresentam um desvio padrão de 0,0975936, média de 0,2455, valor mínimo de 0,048866 e valor máximo de 0,6392344. Considera-se, para fins de análise, a média acrescida de um desvio padrão em que se tem o limite máximo de 0,3431 e a média menos um desvio padrão como o limite mínimo de 0,1479 .

Portanto, em consideração a essa definição dos valores mínimo e máximo, permaneceram os valores acima de 0,1479 e abaixo de 0,3431, o que resultou em 136 observações, que representam a amostra para o presente estudo. Complementarmente, considerou-se a razão produção/custo e determinou-se o valor esperado de 0,2628 como referência para fins de análise dos resultados, com base nos valores determinados pela empresa como padrão para uma equipe de serviço da classe L (1.800 unidades de medida/custo padrão $\mathrm{R} \$ 6.850,00=0,2628)$.

\subsection{Teste de associação}

O teste de associação, segundo Barbetta (2001), verifica se duas ou mais variáveis se apresentam associadas. Assim, pode-se dizer que duas variáveis estão associadas se o conhecimento de uma altera a probabilidade de algum resultado da outra.

Para se estudar a associação entre produtividade e tempo médio da variável experiência, para o período de 24 meses de produção, do caso de estudo, aplicou-se o teste Qui-quadrado. Para tal, após a eliminação dos outliers da amostra, conforme já mencionado, sobraram 136 observações, as quais foram categorizadas conforme a Tabela 3 para as variáveis Produtividade e Experiência.

Tabela 3. Frequência das 136 observações

\begin{tabular}{|c|c|c|c|c|}
\hline \multirow[b]{2}{*}{$\begin{array}{l}\text { Produtividade } \\
\text { (produção/custo) }\end{array}$} & \multicolumn{3}{|c|}{ Experiência } & \multirow[b]{2}{*}{ Total } \\
\hline & $\begin{array}{c}\text { Alta } \\
>425 \mathrm{a} \leq 625 \\
(\%)\end{array}$ & $\begin{array}{c}\text { Média } \\
>224 \mathrm{a} \leq 424 \\
(\%)\end{array}$ & $\begin{array}{c}\text { Baixa } \\
22 \leq 223 \\
(\%)\end{array}$ & \\
\hline $\begin{array}{c}\text { Alta }-0,28 \text { até } 0,34 \\
(\%)\end{array}$ & $\begin{array}{c}5 \\
(50)\end{array}$ & $\begin{array}{c}7 \\
(26,92)\end{array}$ & $\begin{array}{c}28 \\
(26,53)\end{array}$ & $\begin{array}{c}40 \\
(29,41)\end{array}$ \\
\hline $\begin{array}{c}\text { Esperada }-0,22 \text { até } 0,27 \\
(\%)\end{array}$ & $\begin{array}{c}3 \\
(30)\end{array}$ & $\begin{array}{c}7 \\
(26,92)\end{array}$ & $\begin{array}{c}37 \\
(37,76)\end{array}$ & $\begin{array}{c}47 \\
(34,56)\end{array}$ \\
\hline $\begin{array}{c}\text { Baixa }-0,15 \text { até } 0,21 \\
(\%)\end{array}$ & $\begin{array}{c}2 \\
(20)\end{array}$ & $\begin{array}{c}12 \\
(46,16)\end{array}$ & $\begin{array}{c}35 \\
(35,71)\end{array}$ & $\begin{array}{c}49 \\
(36,03)\end{array}$ \\
\hline Total & $\begin{array}{c}10 \\
(100)\end{array}$ & $\begin{array}{c}26 \\
(100)\end{array}$ & $\begin{array}{c}100 \\
(100)\end{array}$ & $\begin{array}{c}136 \\
(100)\end{array}$ \\
\hline
\end{tabular}

Fonte: Dados da pesquisa

Foram categorizados os dados, conforme a Tabela 3, da variável experiência em: Baixa - quando a equipe atingiu uma média de tempo de no mínimo 22 dias e no máximo 223 dias; Média - de 224 a 424 dias; e Alta - de 425 a 625 dias.

Quanto à variável produtividade em: Baixa (conforme valores mínimo e máximo da 
amostra da razão produção em unidades de medidas/custos em $\mathrm{R} \$$ ) - de 0,15 até 0,21 ; Esperada - de 0,22 a 0,27 (levou-se em consideração o padrão de 0,2628 resultante da meta de 1.800 unidades de medida e o custo de $\mathrm{R} \$ 6.850,00$ para a equipe padrão); e Alta a razão de 0,28 a 0,34 .

\subsection{Estatística do teste}

Para a estatística do teste aplicou-se o Qui-quadrado $\left(\chi^{2}\right)$ à medida de distância entre as frequências observadas, representada por $\boldsymbol{O}$, e às frequências que se esperava encontrar em cada célula, representada por $\boldsymbol{E}$, na busca da suposição das variáveis serem independentes.

A frequência esperada é considerada como a distribuição percentual de Produtividade Baixa, Média e Alta em toda a amostra (Tabela 3 - 36,03\% Baixa; 34,56\% Média e 29,41\% Alta). Se Produtividade e Experiência forem variáveis independentes ou não associadas $\left(H_{0}\right)$, então espera-se a manutenção destas percentagens, tanto no estrato da Experiência baixa, média ou alta.

Para o cálculo da frequência esperada utilizou-se a seguinte formula:

$$
E=\frac{(\text { total da linha) } x \text { (total da coluna) }}{\text { (total geral) }}
$$

A Tabela 4 apresenta os valores das células após a aplicação da fórmula.

Tabela 4. Frequência esperada para cada situação

\begin{tabular}{|c|c|c|c|c|}
\hline \multirow[b]{2}{*}{$\begin{array}{l}\text { Produtividade } \\
\text { (produção/custo) }\end{array}$} & \multicolumn{3}{|c|}{ Experiência } & \multirow[b]{2}{*}{ Total } \\
\hline & $\begin{array}{c}\text { Alta } \\
>425 \mathrm{a} \leq 625 \\
(\%)\end{array}$ & $\begin{array}{c}\text { Média } \\
>224 \mathrm{a} \leq 424 \\
(\%)\end{array}$ & $\begin{array}{c}\text { Baixa } \\
22 \leq 223 \\
(\%)\end{array}$ & \\
\hline $\begin{array}{c}\text { Alta }-0,28 \text { até } 0,34 \\
(\%)\end{array}$ & $E=2,94$ & $E=7,65$ & $\mathrm{E}=29,41$ & 40 \\
\hline $\begin{array}{c}\text { Esperada }-0,22 \text { até } 0,27 \\
(\%)\end{array}$ & $\mathrm{E}=3,45$ & $\mathrm{E}=8,99$ & $E=34,56$ & 47 \\
\hline $\begin{array}{c}\text { Baixa }-0,15 \text { até } 0,21 \\
(\%)\end{array}$ & $E=3,60$ & $E=9,37$ & $E=36,03$ & 49 \\
\hline Total & 10 & 26 & 100 & 136 \\
\hline
\end{tabular}

Fonte: Dados da pesquisa

A estatística do teste do Qui-quadrado, segundo Barbetta (2001), é definida por $\chi^{2}=$ $\sum \frac{(O-E)^{2}}{E}$. Diante disso, calculou-se as contribuições do $\chi^{2}$, conforme a Tabela 5 , denominada de tabela de contingência.

Tabela 5. Soma das casas da tabela de contingências

\begin{tabular}{c|c|c|c}
\hline \multirow{2}{*}{$\begin{array}{c}\text { Produtividade } \\
\text { (produção/custo) }\end{array}$} & \multicolumn{3}{|c}{ Experiência } \\
\cline { 2 - 4 } & $\begin{array}{c}\text { Alta } \\
425 \mathrm{a} \leq 625 \\
(\%)\end{array}$ & $\begin{array}{c}\text { Média } \\
>224 \mathrm{a} \leq 424 \\
(\%)\end{array}$ & $\begin{array}{c}\text { Baixa } \\
22 \leq 223 \\
(\%)\end{array}$ \\
\hline $\begin{array}{c}\text { Alta }-0,28 \text { até } 0,34 \\
(\%)\end{array}$ & $\frac{(5-2,94)^{2}}{2,94}=1,4434$ & $\frac{(7-7,65)^{2}}{7,65}=0,0552$ & $\frac{(28-29,41)^{2}}{29,41}=0,0676$ \\
\hline $\begin{array}{c}\text { Esperada }-0,22 \text { até } 0,27 \\
(\%)\end{array}$ & $\frac{(3-3,45)^{2}}{3,45}=0,0587$ & $\frac{(7-8,99)^{2}}{8,99}=0,4405$ & $\frac{(37-34,56)^{2}}{34,56}=0,1723$ \\
\hline $\begin{array}{c}\text { Baixa }-0,15 \text { até } 0,21 \\
(\%)\end{array}$ & $\frac{(2-3,60)^{2}}{3,60}=0,7111$ & $\frac{(12-9,37)^{2}}{9,37}=0,738$ & $\frac{(35-36,03)^{2}}{36,03}=0,0294$ \\
\hline
\end{tabular}

Fonte: Dados da pesquisa

A soma dos valores de cada célula resulta no Qui-quadrado calculado: $\chi^{2}=1,4434+$ 
$0,0552+0,0676+0,0587+0,4405+0,1723+0,7111+0,738+0,0294=\mathbf{3 , 7 1 6 1}$.

Segundo Barbetta (2001), um valor pequeno de $\chi^{2}$ indica que as variáveis podem ser independentes. Nesse caso, não se pode rejeitar a hipótese nula $\left(\mathrm{H}_{0}\right)$, visto não existir associação entre as variáveis Experiência e Produtividade das equipes estudadas ao longo do período para o tempo médio das equipes $x_{P r}=\tilde{x}_{E}$. Ou seja, podem ser meramente casuais. Já, um valor grande pode sinalizar que as diferenças entre frequências observadas e frequências esperadas não devem ser meramente casuais, e que deve haver associação entre as variáveis. Portanto, pode-se rejeitar a hipótese nula e adotar a hipótese alternativa $\left(H_{1}\right)$, de que experiência e produtividade são variáveis relacionadas nas equipes estudadas $x_{\operatorname{Pr}} \neq \tilde{x}_{E}$.

As condições para efetuar o teste estatístico são: a) os dados devem estar dispostos numa tabela de contingência, ou seja, cada elemento observado é alocado apenas numa célula; e b) as amostras devem ser grandes (verificadas em termos de frequência esperada).

\subsection{Distribuição do teste}

Trata-se de uma distribuição Qui-quadrado com $\mathrm{gl}=(1-1)$. $(\mathrm{c}-1)$ graus de liberdade. Então, se $1=3, \mathrm{c}=3$, tem-se que: $\mathrm{gl}=(3-1) .(3-1)=4$. Logo, se $H_{0}$ for verdadeira, os possíveis valores da estatística $\chi^{2}$ devem seguir uma distribuição Qui-quadrado com $\mathrm{gl}=4$ graus de liberdade.

\subsection{Probabilidade de significância}

O valor $p$ representa a probabilidade da estatística $\chi^{2}$ acusar um valor maior ou igual ao valor do $\chi^{2}$ calculado a partir dos dados em análise. Para esta análise atribui-se o nível de significância $\alpha=0,05$, assim se $\mathrm{p}>\alpha$, aceita-se $H_{0}$. Caso contrário, se $\mathrm{p} \leq \alpha$, rejeita-se $H_{0}$.

Observa-se, na Figura 2, com base na saída fornecida pelo pacote estatístico Gretl, que o valor crítico da distribuição Qui-quadrado, considerando-se o nível de significância 0,05 e grau de liberdade 4, corresponde ao valor de 9,48773.

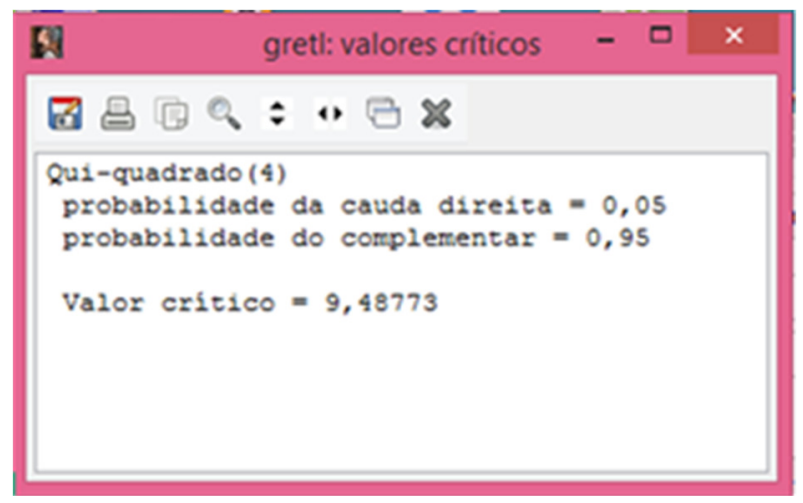

Figura 2. Distribuição Qui-quadrado Fonte: Dados da pesquisa

O valor tabulado de $\chi^{2}=9,48773$ representa a área da cauda direita. Como o valor calculado do $\chi^{2}$ (que corresponde ao valor de 3,7161) está fora dessa área, então não se pode rejeitar $\boldsymbol{H}_{\mathbf{0}}$. 


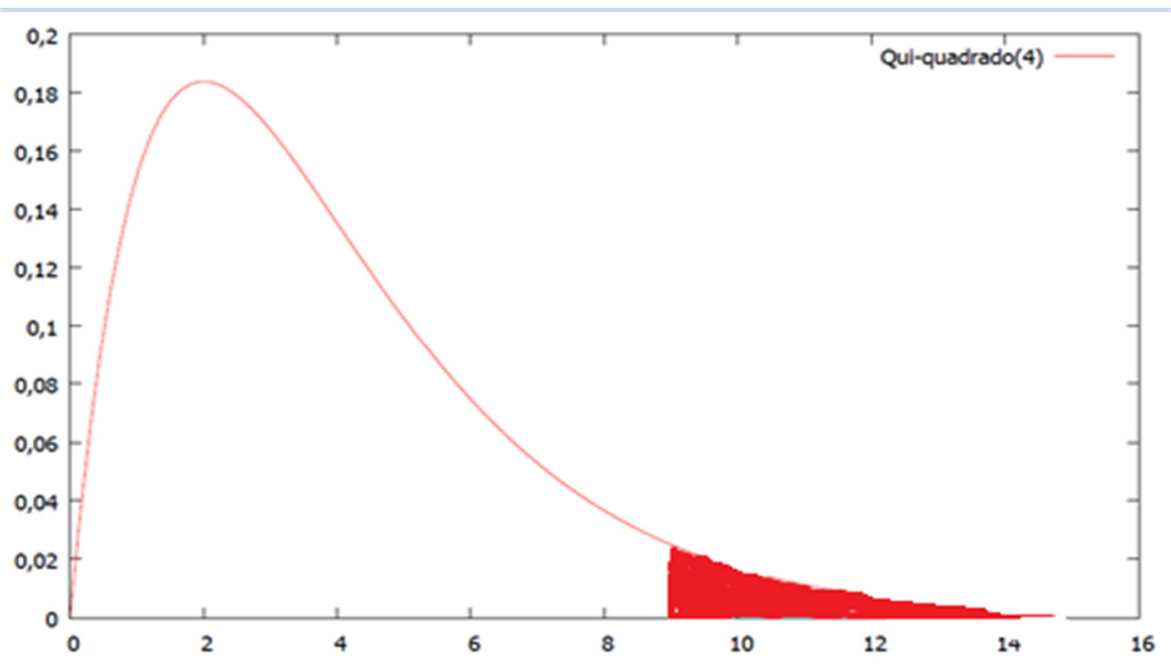

Figura 3. Curva Qui-quadrado

Fonte: Dados da pesquisa

Pelo exposto na Figura 3, pode-se inferir que não há associação entre as variáveis Experiência e Produtividade de acordo com os níveis categorizados e classificados nas 136 observações do caso estudado. Assim, conclui-se que os dados não mostram evidência de associação entre as variáveis das equipes em estudo. A diferença entre a proporção de produtividade alta, esperada e baixa e a proporção de experiência alta, média e baixa pode ser explicada simplesmente por variações casuais.

Tal fato corrobora, em parte, a pesquisa de Wiersma (2007) ao concluir que o aumento da capacidade e a oportunidade de aprender levam aos benefícios esperados com empregados temporários, mas os processos que conduzem a essa aprendizagem não são bem compreendidos. $\mathrm{E}$, no presente estudo há dificuldade para sustentar essa afirmação.

Por fim, vale destacar que os achados estão de acordo com os estudos de Huckman, Staats e Upton (2009), quanto à constatação de que as medidas convencionais da experiência de cada membro da equipe como, por exemplo, anos na empresa, não são consistentemente relacionadas com as performances.

\section{CONCLUSÃO}

O desenvolvimento de estudos para explicar o impacto do fator experiência em empresas prestadoras de serviços foi citado por Boone e Ganeshan (2001) como uma importante lacuna sobre o assunto. Tais empresas caracterizam-se pelo uso intensivo de recursos como a mão de obra na execução de atividades e são organizadas, em geral, por meio de equipes de trabalho. Nessa direção, especificamente, Stroieke, Fogliatto e Anzanello (2013) sugerem estudos com abordagem na modelagem da aprendizagem coletiva, ou de como se comporta tal processo considerando-se a sinergia dos integrantes das equipes.

Assim, sob a indagação de que a repetição das atividades e a permanência do mesmo profissional numa equipe de trabalho pode interferir na relação entre os esforços produtivos (Huckman, Staats, \& Upton, 2009), a presente pesquisa verificou se existe associação entre "experiência coletiva", medida pelo tempo de permanência dos integrantes nas equipes de trabalho, e "produtividade" na prestação de serviços em telecomunicações, de uma empresa que usa um modelo de equivalência para medir quantitativamente a sua diversificada produção. Vale destacar que tal unidade de medida é útil para somar a produção e, assim, possibilitar a associação com outras variáveis que podem influenciar no aumento ou diminuição da produtividade, com consequência para a aprendizagem das equipes (Boone \& Ganeshan, 2001). 
Para medir tal associação, na presente pesquisa, foi utilizado o teste Qui-quadrado, que permite verificar a sua existência ou não. Todavia, como principal resultado, pode-se inferir que os dados utilizados não apontam evidência de associação entre "Experiência" e "Produtividade" das equipes para o caso estudado, dentro dos procedimentos metodológicos aplicados. Assim, a diferença entre a proporção de produtividade alta, esperada e baixa e a proporção de experiência alta, média e baixa pode ser explicada simplesmente por variações casuais.

Paralelamente às proposições sugeridas por Anzanello e Fogliatto (2011) e Stroieke, Fogliatto e Anzanello (2013), acerca de pesquisas em curva de aprendizagem nas organizações, o presente estudo contribui com a literatura no sentido de que explorou o assunto à luz da experiencia coletiva, no referido caso, representada pela formação de equipes homogêneas de trabalho com certo tempo de dedicação, o que atende ao sugerido por Ostermeier (2019), qual seja a busca pelo seu relacionamento com as performances.

Por fim, o presente estudo apresenta algumas limitações que devem ser consideradas ao se interpretar os achados, os quais dizem respeito basicamente ao tamanho da amostra e as variáveis utilizadas para mensuração da aprendizagem.

Sobre o período utilizado, para futuras pesquisas, sugere-se um número maior para verificar a associação das duas variáveis, bem como visitas a campo para fins de acompanhamento, verificação e medição dos tempos de desenvolvimento de cada projeto pelas equipes, com o propósito de identificar outras variáveis que possam interferir na aprendizagem e, assim, implicar de forma positiva ou não na produtividade. E, quanto ao cálculo das médias das equipes, sugere-se a partir dos dados coletados de forma individual aplicar outros modelos como os aplicados pelas curvas de aprendizagem para a obtenção de novos índices de experiência.

\section{REFERÊNCIAS}

Adler, P. S., \& Clark, K. B. (1991). Behind the learning curve: a sketch of the learning process. Management Science, 37(3), 267-281.

Alchian, A. (1963). Reliability of progress curves in airframe production. Econometrica: Journal of the Econometric Society, 31(4), 679-693.

Antonello, C. S., \& Godoy, A. S. (2010). A encruzilhada da aprendizagem organizacional: uma visão multiparadigmática. Revista de Administração Contemporânea, 14(2), 310-332.

Anzanello, M. J., \& Fogliatto, F. S. (2011). Learning curve models and applications: literature review and research directions. International Journal of Industrial Ergonomics, 41(5), 573583.

Argote, L. (1999). Organizational learning: creating, retaining and transferring knowledge. Kluwer Academic Publishers: Boston.

Argote, L., McEvily, B., \& Reagans, R. (2003). Managing knowledge in organizations: an integrative framework and review of emerging themes. Management Science, 49(4), 571582.

Badiru, A. B. (1992). Computational survey of univariate and multivariate learning curve models. Engineering Management, 39(2), 176-188.

Barbetta, P. A. (2008). Estatística aplicada às ciências sociais. Editora da UFSC: Florianópolis.

Boone, T., \& Ganeshan, R. (2001). The effect of information technology on learning in professional service organizations. Journal of Operations Management, 19(4), 485-495. 
Bornia, A. C. (2010). Análise gerencial de custos: aplicação em empresas modernas. São Paulo: Atlas.

Colares, L. G. T. (2005). Processo de trabalho, saúde e qualidade de vida no trabalho em uma unidade de alimentação e nutrição: uma abordagem qualitativa. (Tese de Doutorado). Escola Nacional de Saúde Pública, Fundação Oswaldo Cruz, Rio de Janeiro, RJ.

Conley, P. (1970). Experience curves as a planning tool. Spectrum, 7(6), 63-68.

Corning, P. A. (1983). The synergism hypothesis: on the concept of synergy and its role in the evolution of complex systems. New York: McGraw-Hill Publishers.

Davenport, T. H., \& Prusak, L. (2000). Conhecimento empresarial: como as organizações gerenciam o seu capital intelectual. 2a ed. Rio de Janeiro: Campus.

De La Villarmois, O., \& Levant, Y. (2011). From adoption to use of a management control tool: case study evidence of a costing method. Journal of Applied Accounting Research, 12(3), 234-259.

De La Villarmois, O., \& Levant, Y. (2007). Le Time-Driven ABC: la simplification de l'évaluation des coûts par le recours aux équivalents-un essai de positionnement. Revue Finance Contrôle Stratégie, 10(1), 149-182.

De Wit, M., Junginger, M., Lensink, S., Londo, M., \& Faaij, A. (2010). Competition between biofuels: modeling technological learning and cost reductions over time. Biomass and Bioenergy, 34(2), 203-217.

Demeester, L. L., \& Qi, M. (2005). Managing learning resources for consecutive product generations. International Journal of Production Economics, 95(2), 265-283.

Dutton, J. M., \& Thomas, A. (1984). Treating progress functions as a managerial opportunity. Academy of Management Review, 9(2), 235-247.

Fávero, L. P., Belfiore, P., Silva, F., \& Cham, B. (2009). Análise de dados: modelagem multivariada para tomada de decisão. São Paulo: Elsevier.

Ferrari, M. J. (2012). Custeio de serviços baseado em unidade de medida de produção: o caso de uma empresa do setor de telecomunicações. (Dissertação de Mestrado). Programa de Pós-Graduação em Contabilidade, Universidade Federal de Santa Catarina, Florianópolis, SC.

Fioretti, G. (2007). The organizational learning curves. European Journal of Operational Research, 177(3), 1375-1384.

Gervais, M., \& Levant, Y. (2007). Comment garantir l'homogénéité globale dans la méthode UVA? Deux études de cas". Finance, Contrôle, Stratégie, 10(3), 43-73.

Glock, C. H., Grosse, E. H., Jaber, M. Y., \& Smunt, T. L. (2019). Applications of learning curves in production and operations management: a systematic literature review. Computers \& Industrial Engineering, 131, 422-441.

Horngren, C. T., Datar, S. M., \& Foster, G. (2004). Contabilidade de custos: uma abordagem gerencial. 11a ed. São Paulo: Prentice Hall.

Huckman, R. S., Staats, B. R., \& Upton, D. M. (2009). Team familiarity, role experience, and performance: evidence from Indian software services. Management science, 55(1), 85-100.

Jaber, M. Y., \& Bonney, M. (1996). Optimal lot sizing under learning considerations: the bounded learning case. Applied Mathematical Modelling, 20(10), 750-755. 
Kahouli-Brahmi, S. (2008). Technological learning in energy-environment-economy modelling: a survey. Energy Policy, 36(1), 138-162.

Leite, M. O. (2002). A utilização das curvas de aprendizagem no planejamento da construção civil. (Dissertação de Mestrado). Programa de Pós-Graduação em Engenharia de Produção, Universidade Federal de Santa Catarina, Florianópolis, SC.

Levant, Y., \& Zimnovitch, H. (2013). Contemporary evolutions in costing methods: understanding these trends through the use of equivalence methods in France. Accounting History, 18(1), 51-75.

Lohmann, M., Anzanello, M. J., Fogliatto, F. S., \& Da Silveira, G. C. (2019). Grouping workers with similar learning profiles in mass customization production lines. Computers \& Industrial Engineering, 131, 542-551.

Morrison, J. B. (2008). Putting the learning curve in context. Journal of Business Research, 61(11), 1182-1190.

Nadeau, M. C., Kar, A., Roth, R., \& Kirchain, R. (2010). A dynamic process-based cost modeling approach to understand learning effects in manufacturing. International Journal of Production Economics, 128(1), 223-234.

Nemet, G. F. (2006). Beyond the learning curve: factors influencing cost reductions in photovoltaics. Energy policy, 34(17), 3218-3232.

Neuhaus, M., \& Bunke, H. (2007). Automatic learning of cost functions for graph edit distance. Information Sciences, 177(1), 239-247.

Ostermeier, F. F. (2019). The impact of human consideration, schedule types and product mix on scheduling objectives for unpaced mixed-model assembly lines. International Journal of Production Research, 57, 1-20.

Pereira, W. C., \& Suslick, S. B. (2003). Modelo de previsão do custo de mineração pelo sistema de curvas de aprendizado. Revista Escola de Minas, 56(2), 139-143.

Plaza, M., \& Rohlf, K. (2008). Learning and performance in ERP implementation projects: a learning-curve model for analyzing and managing consulting costs. International Journal of Production Economics, 115(1), 72-85.

Reis, L., Borgert, A., \& Ferrari, M. J. (2016). Unidade de equivalência em serviços de telecomunicações. Gestão da Produção, Operações e Sistemas, 11(1).

Schoots, K., Kramer, G. J., \& Van Der Zwaan, B. C. C. (2010). Technology learning for fuel cells: an assessment of past and potential cost reductions. Energy Policy, 38(6), 2887-2897.

Smunt, T. L., \& Watts, C. A. (2003). Improving operations planning with learning curves: overcoming the pitfalls of 'messy' shop floor data. Journal of Operations Management, 21(1), 93-107.

Stroieke, E., Fogliatto, F. S., \& Anzanello, M. J. (2013). Estado da arte das aplicações de curvas de aprendizado. Gestão \& Produção, 20(3), 681-694.

Sveiby, K. E. (1998). A nova riqueza das organizações: gerenciando e avaliando patrimônios de conhecimento. Rio de Janeiro: Campus.

Szwarcfiter, C., \& Dalcol, P. R. T. (1997). Economias de escala e de escopo: desmistificando alguns aspectos da transição. Production, 7(2), 117-129.

Teplitz, C. J. (1991). The learning curve deskbook: a reference guide to theory, calculations, and applications. New York: Quorum Books. 
Togo, D. F. (2001). A curvilinear approach to teaching learning effects for cost estimation. Journal of Accounting Education, 19(3), 211-223.

Tsuchiya, H., \& Kobayashi, O. (2004). Mass production cost of PEM fuel cell by learning curve. International Journal of Hydrogen Energy, 29(10), 985-990.

Van Den Broek, M., Hoefnagels, R., Rubin, E., Turkenburg, W., \& Faaij, A. (2009). Effects of technological learning on future cost and performance of power plants with $\mathrm{CO} 2$ capture. Progress in Energy and Combustion Science, 35(6), 457-480.

Van Den Wall Bake, J. D., Junginger, M., Faaij, A., Poot, T., \& Walter, A. (2009). Explaining the experience curve: cost reductions of Brazilian ethanol from sugarcane. Biomass and Bioenergy, 33(4), 644-658.

Weitzel, L., Rovere, E. L., \& Cunha, R. (2006). As demandas do novo modelo institucional do setor elétrico brasileiro. Anais do Congresso Brasileiro de Planejamento Energético, Brasília, DF, 5.

Wiersma, E. (2007). Conditions that shape the learning curve: factors that increase the ability and opportunity to learn. Management Science, 53(12), 1903-1915.

Wright, T. P. (1936). Factors affecting the cost of airplanes. Journal of the aeronautical sciences, 3(4), 122-128.

Young, W. A., Masel, D. T., \& Judd, R. P. (2008). A matrix-based methodology for determining a part family's learning rate. Computers \& Industrial Engineering, 54(3), 390-400. 